\title{
PROPOSTA PARA PADRONIZAC̣ÃO dO RELATÓRIO DE TOMOGRAFIA COMPUTADORIZADA NOS ANEURISMAS DA AORTA ABDOMINAL*
}

\author{
Fabiola Goda Torlai ${ }^{1}$, Gustavo S. Portes Meirelles ${ }^{1}$, Fausto Miranda $\mathrm{Jr}^{2}{ }^{2}$, José Honório \\ A.P. da Fonseca ${ }^{3}$, Sérgio Ajzen ${ }^{4}$, Giuseppe D'Ippolito ${ }^{5}$
}

Resumo OBJETIVO: Propor um modelo de padronização de relatório para aneurisma da aorta abdominal na tomografia computadorizada. MATERIAIS E MÉTODOS: Foram realizadas, no período de abril a outubro de 2004, entrevistas com integrantes da Disciplina de Cirurgia Vascular da nossa instituição, para elaboração de um modelo de padronização de relatório de tomografia computadorizada para o estudo do aneurisma da aorta abdominal. A partir deste modelo foi elaborado um questionário, enviado a nove outros cirurgiões, todos com experiência em cirurgia da aorta abdominal. $O$ índice de resposta aos questionários foi de 55,5\% (5/9). RESULTADOS: Os parâmetros de interesse citados mais freqüentemente para a avaliação dos aneurismas de aorta abdominal foram: diâmetro máximo do colo proximal, extensão do colo proximal até a artéria renal mais baixa, forma do colo proximal, diâmetro máximo do aneurisma e diâmetro das artérias ilíacas comuns. Estes dados permitiram elaborar uma proposta de modelo para padronização de relatório na tomografia computadorizada. CONCLUSÃO: Um modelo para a análise tomográfica padronizada do aneurisma de aorta abdominal permite atender às necessidades dos cirurgiões vasculares para acompanhar a evolução e planejar o tratamento destes pacientes.

Unitermos: Aorta; Aneurisma; Tomografia computadorizada.

Abstract A proposal for standardizing computed tomography reports on abdominal aortic aneurysms.

OBJECTIVE: To propose a model to standardize computed tomography reports on abdominal aortic aneurysms. MATERIALS AND METHODS: Interviews were carried out with members of Vascular Surgery Division of our institution, in the period between April and October 2004, aiming at developing a standardized model of computed tomography reports on abdominal aortic aneurysms. Based on this model, a questionnaire was elaborated and sent to other nine surgeons, all of them experienced in the field of abdominal aortic surgery. The questionnaires response rate was $55.5 \%(5 / 9)$. RESULTS: The most frequently mentioned parameters of interest for evaluation of abdominal aortic aneurysms were: maximum diameter of proximal aortic neck, proximal aortic neck length to lower renal arteries, shape of proximal aortic neck, maximum diameter of the aneurysm and diameter of the common iliac arteries. These data allowed the development of a proposal for a model to standardize computed tomography reports. CONCLUSION: A model for standardized tomographic analysis of abdominal aortic aneurysms has met vascular surgeons' needs for following-up patients and planning their treatment.

Keywords: Aorta; Aneurysm; Computed tomography.

\section{INTRODUÇÃO}

O aneurisma da aorta é definido como uma área de dilatação permanente na qual

* Trabalho realizado na Universidade Federal de São Paulo Escola Paulista de Medicina, São Paulo, SP.

1. Médicos Radiologistas.

2. Professor Livre-Docente do Departamento de Cirurgia, Disciplina de Cirurgia Vascular da Universidade Federal de São PauloEscola Paulista de Medicina.

3. Professor Livre-Docente do Departamento de Cirurgia, Disciplina de Cirurgia Cardiovascular da Universidade Federal de São Paulo-Escola Paulista de Medicina.

4. Professor Adjunto Livre-Docente do Departamento de Diagnóstico por Imagem da Universidade Federal de São Paulo-Escola Paulista de Medicina.

5. Professor Adjunto do Departamento de Diagnóstico por Imagem da Universidade Federal de São Paulo-Escola Paulista de Medicina.

Endereço para correspondência: Prof. Dr. Giuseppe D'Ippolito. Rua Professor Filadelfo Azevedo, 617, ap. 61, Vila Nova Conceição. São Paulo, SP, 04508-001. E-mail: giuseppe_dr@uol. com.br

Recebido para publicação em 1/11/2005. Aceito, após revisão, em 28/11/2005. o diâmetro máximo é pelo menos $50 \%$ maior que a aorta normal ou o segmento normal logo acima do aneurisma ${ }^{(\mathbf{1})}$. Existem referências, na literatura, que consideram o aneurisma de aorta abdominal como uma dilatação maior que $3 \mathrm{~cm}$ no seu maior diâmetro verdadeiro ${ }^{(2)}$.

Os fatores de risco para o aneurisma da aorta abdominal são os mesmos da aterosclerose: idade maior que 50 anos, sexo masculino, tabagismo e doença pulmonar obstrutiva crônica ${ }^{(2)}$.

Os aneurismas da aorta abdominal possuem um índice de expansão que varia de 2-4 mm por ano para aneurismas menores que $4 \mathrm{~cm}$, de $2-5 \mathrm{~mm}$ por ano para aneurismas entre $4-5 \mathrm{~cm}$ e de $3-7 \mathrm{~mm}$ para aneurismas maiores que $5 \mathrm{~cm}$. $\mathrm{O}$ risco de ruptura em quatro anos é de $2 \%$ para aneu- rismas menores que $4 \mathrm{~cm}, 10 \%$ para aneurismas entre $4-5 \mathrm{~cm}$ e $22 \%$ para aneurismas maiores que $5 \mathrm{~cm}^{(3,4)}$.

Estudos têm demonstrado um aumento na incidência do aneurisma de aorta. Dos anos 70 para os anos 90 a incidência dobrou, impulsionada pela melhoria nos métodos de imagem, principalmente pelo advento da tomografia computadorizada (TC) e também pelo aumento na expectativa de vida da população ${ }^{(5)}$.

O objetivo do tratamento do aneurisma de aorta consiste em excluí-lo da pressão sanguínea arterial, com preservação do fluxo sanguíneo através do implante de um conduto vascular, eliminando o risco de ruptura e morte ${ }^{(\mathbf{4 , 6})}$.

A relevância dos fatores anatômicos na determinação da conduta do paciente faz 
com que os exames de imagem sejam fundamentais na avaliação do aneurisma de aorta, especialmente através de relatórios bem padronizados e de fácil compreensão ${ }^{(4,7)}$.

O nosso objetivo foi o de propor um esquema de padronização de relatório para o estudo de aneurismas de aorta abdominal por meio da TC.

\section{MATERIAIS E MÉTODOS}

Foram realizadas entrevistas com 14 cirurgiões no período de abril a outubro de 2004. Primeiramente, foram entrevistados cinco docentes da Disciplina de Cirurgia Vascular da Universidade Federal de São Paulo-Escola Paulista de Medicina (Unifesp-EPM), com familiaridade sobre o assunto, para discutirmos o modelo de relatório tomográfico para o estudo de aneurismas da aorta abdominal. Formulamos então a seguinte pergunta: “Quais parâmetros o senhor julga importantes e que deveriam constar num relatório de TC de um paciente com aneurisma de aorta abdominal?".

Elaboramos em seguida um modelo de laudo tomográfico baseado nas opiniões desses primeiros entrevistados, que foi então encaminhado por correio eletrônico, através de questionários digitais, para outros nove cirurgiões que lidam com aneurismas de aorta, sem vínculos com a Unifesp-EPM, indicados por colegas desta universidade. O índice de resposta aos questionários foi de 55,5\% (5/9).

A partir das entrevistas realizadas na Unifesp-EPM e das respostas aos questionários eletrônicos, procedemos à análise estatística dos dados e à formulação do modelo final de relatório para aneurisma de aorta abdominal.

\section{RESULTADOS}

Os resultados obtidos estão organizados na Tabela 1. Houve consenso entre os entrevistados quanto à importância de descrever, no relatório, medidas relacionadas ao diâmetro do colo proximal do aneurisma, extensão do colo proximal até a artéria renal mais baixa, forma do colo proximal, diâmetro máximo da aorta e das artérias ilíacas comuns. O diâmetro da luz da aorta e permeabilidade das artérias ilíacas internas não mereceu atenção por parte da maioria dos entrevistados.

$\mathrm{Na}$ Figura 1 apresentamos um modelo sugerido para o padrão de relatório tomográfico de análise do aneurisma da aorta abdominal.

\section{DISCUSSÃO}

O primeiro modelo de relatório para aneurismas da aorta foi publicado em 1997 pelo Comitê de Padronização de Relatórios em Cirurgia Vascular da Sociedade de Ci-

Tabela 1 Parâmetros para relatório de tomografia computadorizada de aneurismas da aorta abdominal.

\begin{tabular}{|l|c|}
\hline Parâmetros para aorta abdominal & Respostas dos cirurgiões \\
\hline Diâmetro máximo do colo proximal (D1) & $10 / 10(100 \%)$ \\
Extensão do colo proximal até a artéria renal mais baixa (L1) & $10 / 10(100 \%)$ \\
Forma do colo proximal (reto/tortuoso) & $10 / 10(100 \%)$ \\
Diâmetro máximo do aneurisma (D2) & $10 / 10(100 \%)$ \\
Extensão do aneurisma no maior eixo (L2) & $9 / 10(90 \%)$ \\
Trombos e calcificações na aorta & $7 / 10(70 \%)$ \\
Diâmetro das artérias ilíacas comuns (D3 e D4) & $10 / 10(100 \%)$ \\
Extensão das artérias ilíacas comuns (L3 e L4) & $9 / 10(90 \%)$ \\
Diâmetro das artérias ilíacas externas (D5 e D6) & $6 / 10(60 \%)$ \\
Perviedade e calcificações das artérias ilíacas comuns & $9 / 10(90 \%)$ \\
Tortuosidade das artérias ilíacas comuns & $3 / 10(30 \%)$ \\
Perviedade das artérias ilíacas internas & $1 / 10(10 \%)$ \\
Presença e diâmetro máximo do colo distal & $3 / 10(30 \%)$ \\
Diâmetro da luz da aorta & $1 / 10(10 \%)$ \\
\hline
\end{tabular}

ANEURISMA DE AORTA ABDOMINAL
1) Diâmetro máximo do colo proximal (D1)
2) Extensão colo proximal até a artéria renal mais baixa (L1)
3) Forma do colo proximal (reto/tortuoso)
4) Diâmetro máximo do aneurisma (D2)
5) Extensão do aneurisma no maior eixo (L2)
6) Trombos e calcificações na aorta
7) Diâmetro das artérias ilíacas comuns (D3 e D4)
8) Extensão das artérias ilíacas comuns (L3 e L4)
9) Diâmetro das artérias ilíacas externas (D5 e D6)
10) Penviedade e calcificações das artérias ilíacas comuns
11) Tortuosidade das artérias ilíacas comuns
12) Penviedade das artérias ilíacas internas
13) Presença e diâmetro máximo do colo distal
14) Diâmetro da luz da aorta __a

Figura 1. Modelo de padronização de relatório de tomografia computadorizada para aneurisma de aorta abdominal. 
rurgia Vascular/Sociedade Internacional de Cirurgia Cardiovascular, para aneurismas da aorta abdominal infra-renal, abrangendo fatores clínicos e anatômicos, com base em exames de angiografia, TC e ressonância magnética $(\mathrm{RM})^{(\mathbf{8})}$.

Em 2002, o Comitê de Padronização de Relatórios em Cirurgia Vascular publicou uma atualização, fixando primordialmente os critérios para análise pós-procedimento endovascular. Este consenso recomenda que os aneurismas sejam classificados quanto a localização, etiologia e manifestações clínicas ${ }^{(6)}$.

Outros autores estudaram índices de risco anatômico para a correção endovascular do aneurisma de aorta abdominal, classificando parâmetros como diâmetro, extensão e tortuosidade dos colos proximal e distal; tortuosidade e vasos que se originam do saco aneurismático; perfusão pélvica; diâmetro das artérias ilíacas e sua tortuosidade, entre outros ${ }^{(\mathbf{9 , 1 0})}$.

Alguns reparos anatômicos devem ser definidos para a análise sistemática do aneurisma de aorta. O colo é definido como a região da aorta com diâmetro normal logo acima ou abaixo do aneurisma, sendo o local de conexão da prótese à aorta, onde deve estar efetivamente fixada e selada ${ }^{(11)}$. O colo distal pode ou não estar presente, podendo o aneurisma se estender para as artérias ilíacas. A medida da extensão do colo proximal deve ser feita em relação à artéria renal mais distal, e do colo distal em

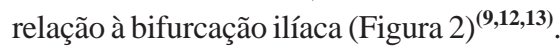

O diâmetro do colo do aneurisma deve ser medido perpendicular ao fluxo sanguíneo. Quando este não é perpendicular ao plano de corte, a menor medida do diâmetro em cortes elípticos é considerada uma aproximação apropriada da medida do diâmetro verdadeiro ${ }^{(\mathbf{4})}$. Alguns autores consideram a medida da angulação do colo um fator importante na análise do aneurisma de aorta $^{(9)}$, e este parâmetro foi também valorizado pelos médicos por nós entrevistados.

O diâmetro máximo do aneurisma é um dos fatores mais importantes no estudo do aneurisma de aorta, devido ao risco de ruptura estar relacionado ao tamanho e ao seu índice de expansão ${ }^{(4)}$. $\mathrm{O}$ diâmetro máximo do aneurisma deve ser medido de parede a parede, perpendicular ao fluxo sanguíneo ou ao plano de corte em reconstruções tridimensionais (Figura 2). A menor medida do diâmetro em cortes elípticos é considerada uma aproximação apropriada da medida do diâmetro verdadeiro ${ }^{(\mathbf{6})}$.

Embora não tenha sido valorizado pelos entrevistados, o diâmetro do colo distal do aneurisma, quando presente, também é importante para a escolha do tipo de endoprótese a ser empregada, se bifurcada ou se mono-ilíaca.

A tortuosidade do aneurisma é outro fator de interesse na avaliação anatômica do aneurisma de aorta. Há na literatura diversos métodos para a sua graduação ${ }^{(\mathbf{8 , 9 , 1 4})}$. A presença de trombos no aneurisma também deve ser analisada. Alguns autores classificam a quantidade de trombos atra- vés da área ocupada. Os trombos, juntamente com a tortuosidade do aneurisma e sua angulação, podem influenciar no risco de embolização periprocedimento nos pacientes submetidos à colocação de endopróteses $^{(\mathbf{9})}$, e por esta razão deve-se informar o diâmetro da luz do aneurisma.

A presença de vasos patentes se originando do saco aneurismático também pode influenciar no sucesso do procedimento endovascular, sendo considerados entre os fatores de risco para a colocação de endoprótese $^{(\mathbf{9 , 1 5})}$.

A anatomia das artérias ilíacas no tratamento endovascular dos aneurismas de aorta está relacionada ao acesso para o procedimento, à perfusão para a pelve e órgãos adjacentes nos aneurismas de aorta abdominal e à presença de um ponto de fixação para a endoprótese ${ }^{(\mathbf{9 , 1 3})}$. As complicações relacionadas à anatomia difícil das artérias ilíacas são descritas em $47 \%$ dos pacientes $^{(\mathbf{1 6})}$ e isto explica a importância de informar o calibre, permeabilidade e extensão destes vasos (L3, L4, D3 e D4).

Finalmente, é interessante observar que um modelo semelhante de relatório pode também ser adotado em exames de angioRM (Figura 3), excluindo-se a análise da presença de calcificações parietais, dificilmente detectadas pelo método.

\section{CONCLUSÃO}

Através da análise dos resultados obtidos a partir de entrevistas e questionários

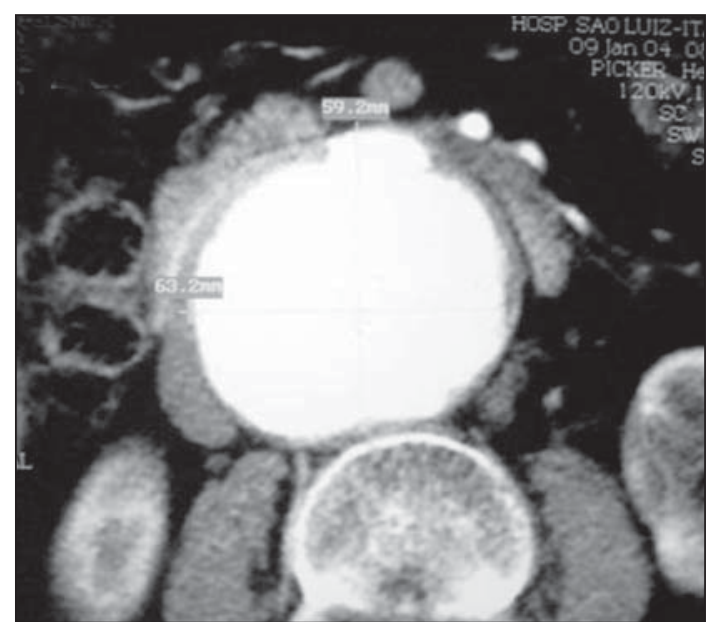

A proximal e distal (setas).

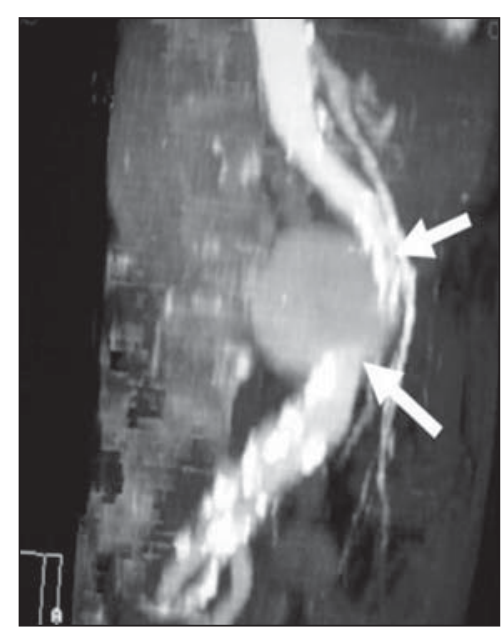

B

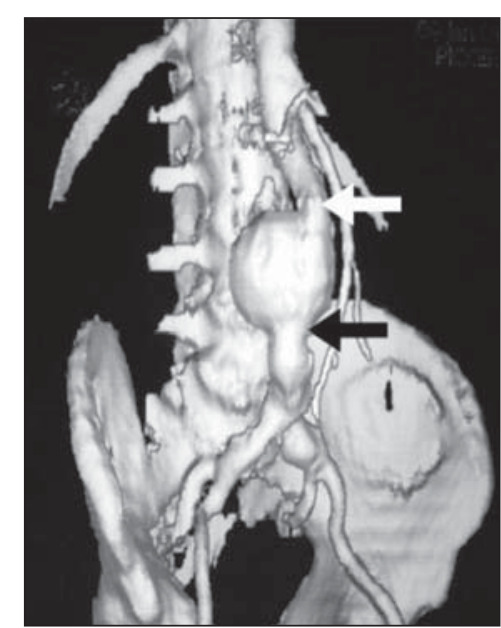

C 


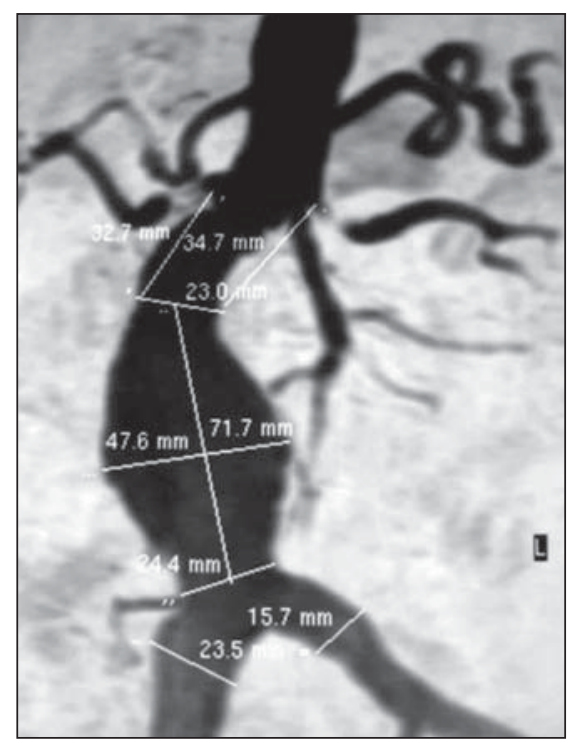

Figura 3. Angio-RM com contraste da aorta abdominal. Medidas do aneurisma da aorta, em relação às artérias renais, bifurcação da aorta e colo proximal e distal do aneurisma. Notar medidas de diâmetro das artérias ilíacas comuns.

respondidos por cirurgiões diretamente envolvidos no tratamento de aneurisma da aorta abdominal, podemos concluir que é possível estabelecer um padrão de relatório de utilidade prática e feito sob medida para este grupo de pacientes e de especialistas.

\section{REFERÊNCIAS}

1. Nguyen BT. Computed tomography diagnosis of thoracic aortic aneurysms. Semin Roentgenol 2001;36:309-324.

2. Kaufman JA, Geller SC, Brewster DC, et al. Endovascular repair of abdominal aortic aneurysms: current status and future directions. AJR Am J Roentgenol 2000;175:289-302.

3. Macura KJ, Corl FM, Fishman EK, Bluemke DA. Pathogenesis in acute aortic syndromes: aortic aneurysm leak and rupture and traumatic aortic transection. AJR Am J Roentgenol 2003;181: 303-307.

4. Masuda Y, Takanashi K, Takasu J, Morooka N Inagaki Y. Expansion rate of thoracic aortic aneurysms and influencing factors. Chest 1992;102: 461-466.

5. Bergeron P, De Chaumaray T, Gay J, Douillez V. Endovascular treatment of thoracic aortic aneurysms. J Cardiovasc Surg (Torino) 2003;44:349 361.

6. Chaikof EL, Blankensteijn JD, Harris PL, et al. Reporting standards for endovascular aortic aneurysm repair. J Vasc Surg 2002;35:1048-1060.

7. Matravers P, Morgan R, Belli A. The use of stent grafts for the treatment of aneurysms and dissections of the thoracic aorta: a single centre experience. Eur J Vasc Endovasc Surg 2003;26:587595.

8. Ahn SS, Rutherford RB, Johnston KW, et al. Reporting standards for infrarenal endovascular abdominal aortic aneurysm repair. Ad Hoc Committee for Standardized Reporting Practices in
Vascular Surgery of The Society for Vascular Surgery/International Society for Cardiovascular Surgery. J Vasc Surg 1997;25:405-410.

9. Chaikof EL, Fillinger MF, Matsumura JS, et al. Identifying and grading factors that modify the outcome of endovascular aortic aneurysm repair. J Vasc Surg 2002;35:1061-1066.

10. Carpenter JP, Baum RA, Barker CF, et al. Impact of exclusion criteria on patient selection for endovascular abdominal aortic aneurysm repair. J Vasc Surg 2001;34:1050-1054.

11. Greenberg RK. Abdominal aortic endografting: fixation and sealing. J Am Coll Surg 2002;194(1 Suppl):S79-87.

12. Rodrigues Alves CM, da Fonseca JH, de Souza JA, Camargo Carvalho AC, Buffolo E. Endovascular treatment of thoracic disease: patient selection and a proposal of a risk score. Ann Thorac Surg 2002;73:1143-1148.

13. Filis KA, Arko FR, Rubin GD, Zarins CK. Threedimensional CT evaluation for endovascular abdominal aortic aneurysm repair. Quantitative assessment of the infrarenal aortic neck. Acta Chir Belg 2003;103:81-86

14. Wolf YG, Tillich M, Lee WA, Rubin GD, Fogarty TJ, Zarins CK. Impact of aortoiliac tortuosity on endovascular repair of abdominal aortic aneurysms: evaluation of 3D computer-based assessment. J Vasc Surg 2001;34:594-599.

15. Gorich J, Rilinger N, Sokiranski R, et al. Endoleaks after endovascular repair of aortic aneurysm: are they predictable? - initial results. Radiology 2001;218:477-480.

16. Henretta JP, Karch LA, Hodgson KJ, et al. Special iliac artery considerations during aneurysm endografting. Am J Surg 1999;178:212-218. 